\title{
Vertex-Neighbor-Scattering Number of Trees
}

\author{
Zongtian Wei ${ }^{1,2}$, Yong Liu ${ }^{2}$, Anchan Mai $^{3}$ \\ ${ }^{1}$ Department of Applied Mathematics, Northwestern Polytechnical University, Xi' an, China \\ ${ }^{2}$ School of Science, Xi' an University of Architecture and Technology, Xi' an, China \\ ${ }^{3}$ Science-Cultural Institute, Xi' an Military Academy, Xi' an, China \\ E-mail:wzt6481@163.com,xianliuyong@126.com,anchan.mai@eyou.com \\ Received April 29, 2011; revised May 15, 2011; accepted May 25, 2011
}

\begin{abstract}
A vertex subversion strategy of a graph $G=(V, E)$ is a set of vertices $S \subseteq V(G)$ whose closed neighborhood is deleted from $G$. The survival subgraph is denoted by $G / S$. We call $S$ a cut-strategy of $G$ if $G / S$ is disconnected, or is a clique, or is $\phi$. The vertex-neighbor-scattering number of $G$ is defined to be $V N S(G)=\max _{S \subseteq V(G)}\{\omega(G / S)-|S|\}$, where $S$ is any cut-strategy of $G$, and $\omega(G / G)$ is the number of the components of $G / S$. It has been proved that the computing problem of this parameter is $N P$-complete, so we discuss the properties of vertex-neighbor-scattering number of trees in this paper.
\end{abstract}

Keywords: Vertex-Neighbor-Scattering Number, Tree, Path, Star, Comet

\section{Introduction}

In [1] we introduced a new graph parameter called "vertex-neighbor-scattering number." We motivate the use of this parameter by viewing a graph as a model of a spy network whose vertices represent agents and whose edges represent lines of communication. If a spy is discovered, the espionage agency can no longer trust any of the spies with whom he was in direct communication. It has been shown that the parameter can measure the "neighbor" stability of graphs [1]. As many graphic parameters, the computing problem of this parameter is $N P$ - complete [2]. So we discuss the properties of the vertex-neighbor-scattering number of trees in this paper. Our definitions follow [3].

Let $G=(V, E)$ be a graph and $u$ a vertex in $G$. $N(u)=\{v \in V(G) \mid u \neq v, u$ and $v$ are adjacent $\}$ is the open neighborhood of $u$, and $N[u]=N(u) \cup\{u\}$ is the closed neighborhood of $u$. A vertex $u$ in $G$ is said to be subverted if its closed neighborhood $N[u]$ is deleted from $G$. A set of vertices $S \subseteq V(G)$ is called a vertex subversion strategy of $G$ if each of the vertices in $S$ has been subverted from $G$. By $G / S$ we denote the survival subgraph left after each vertex of $S$ has been subverted from $G . S$ is called a cutstrategy of $G$ if the survival subgraph $G / S$ is disconnected, or is a clique, or is $\phi$.
The vertex-neighbor-scattering number (VNS) of a connected noncomplete graph $G$ is defined as $V N S(G)=\max _{S \subseteq V(G)}\{\omega(G / S)-|S|\}$, where $S$ is any cutstrategy of $G$, and $\omega(G / S)$ is the number of the components of $G / S$. We call $S^{*} \subseteq V(G)$ a $V N S-$ set of $G$ if $V N S(G)=\omega\left(G / S^{*}\right)-\left|S^{*}\right|$. In particular, we define the vertex-neighbor-scattering number of a complete graph $K_{n}$ to be 1 .

A comet $C_{t, r}$ is a graph obtained by identifying one end of a path $P_{t}(\geq 2)$ with the center of a star $S_{1, r}(r \geq 2)$. The center of $S_{1, r}$ is called the center of $C_{t, r}$.

The following lemmata will be used in the next section.

Theorem 1: [1] Let $P_{n}$ be a path with order $n(\geq 3)$. Then

$$
\operatorname{VNS}\left(P_{n}\right)=\left\{\begin{array}{lll}
0, & \text { if } n=3,4 \\
1, & \text { if } n \geq 5
\end{array}\right.
$$

Theorem 2: [1] Let $S_{1, r}(r \geq 2)$ be a star. Then $\operatorname{VNS}\left(S_{1, r}\right)=r-2$.

Theorem 3: [1] Let $C_{t, r}$ be a comet, both $t$ and $r$ are at least 2. Then 


$$
V N S\left(C_{t, r}\right)= \begin{cases}r-1, & \text { if } t=2,3 \\ r, & \text { if } t \geq 4\end{cases}
$$

\section{Vertex-Neighbor-Scattering Number of Trees}

Theorem 4: Let $T$ be a tree with order $n(\geq 5)$. Then $1 \leq \operatorname{VNS}(T) \leq n-3$.

Proof. 1) For any vertex $v \in V(T),|N[v]| \geq 2$, so $\omega(T / S) \leq n-2$. On the other hand, $T$ is connected and with order $n \geq 5$, then for its any $V N S-\operatorname{set} S$, $|S| \geq 1$ and $\omega(T / S) \leq n-2$. Thus we have

$$
V N S(T)=\max _{S \subseteq V(T)}\{\omega(T / S)-|S|\} \leq n-2-1=n-3
$$

2) We distinguish two cases to prove $\operatorname{VNS}(T) \geq 1$.

Case 1: $T \cong P_{n}$. Since $n \geq 5$, there must exist a vertex $v \in V(T)$ such that $\omega(T /\{v\})=2$. So

$$
\begin{aligned}
V N S(T) & =\max _{S \subseteq V(T)}\{\omega(T / S)-|S|\} \\
& \geq \omega(T /\{v\})-|\{v\}|=2-1=1
\end{aligned}
$$

Case 2: $T \neq P_{n}$. Then there exist at least one vertex in $T$, say $v$, such that $d(v) \geq 3$. Let $u$ be any vertex adjacent to $v$. Then $\omega(T) /\{u\} \geq 2$, this means

$$
\operatorname{VNS}(T) \geq \omega(T) /\{u\} \geq 2-|\{u\}|=2-1=1
$$

Combing Case 1 and Case 2, we have $\operatorname{VNS}(T) \geq 1$. Thus the proof is completed.

Remark 1: When $n=2,3$, the trees with order $n$ are $n=2,3$ or $P_{3}$, respectively. So $\operatorname{VNS}(T)=\operatorname{VNS}\left(P_{n}\right)$. When $n=4$, there are two different trees with order $n$ in isomorphism sense, i.e., $P_{4}$ and $S_{1,3}$. By Theorem 1 and Theorem 2, $\operatorname{VNS}\left(P_{4}\right)=0$, $\operatorname{VNS}\left(S_{1,3}\right)=1$.

Remark 2: The lower and upper bound in Theorem 4 is the best possible, it can be shown by paths and stars, respectively.

Theorem 5: If $l$ is any integer, where $1 \leq l \leq n-3$, then there is a tree $T$ of order $n$ such that $\operatorname{VNS}(T)=l$.

Proof. If $n=4$, then $l=1$. By Theorem 3, $C_{2,2}$ is a tree of order 4 satisfying $\operatorname{VNS}\left(C_{2,2}\right)=1$, the conclusion holds.

Now we assume $n \geq 5$ and distinguish two cases.
Case 1: $l=1$ or $n-3$. If $n-3$, by Theorem 1, $P_{n}$ is a tree of order $n$ satisfying $\operatorname{VNS}\left(P_{n}\right)=1$; if $l=n-3$, by Theorem 2, $S_{1, n-1}$ is a tree of order $n$ satisfying $\operatorname{VNS}\left(S_{1, n-1}\right)=n-3$, the conclusion holds.

Case 2: $1<l<n-3$. Then $n-l \geq 4$. By Theorem 3, $C_{n-l, l}$ is a tree satisfying $\operatorname{VNS}\left(C_{n-l, l}\right)=l$, the conclusion holds.

Theorem 6: 1) When $n \geq 5, S_{1, n-1}$ is the unique tree $T$ with order $n$ such that $\operatorname{VNS}(T)=n-3$.

2) When $n \geq 7, P_{n}$ is the unique tree $T$ with order $n$ such that $\operatorname{VNS}(T)=n-3$.

Proof. 1) Let $T$ be a tree with order $n$ such that $V N S(T)=n-3$. Assume $S$ is an $V N S-$ set of $T$, then $T$. Otherwise, if $|S| \geq 2$, then $|V(T / S)| \leq n-3$, so $\omega(T / S) \leq n-3$ and $V N S(T) \leq \omega(T / S)-|S|<n-3$, a contradiction.

Let $\{v\}$ be an $V N S-$ set of $T$. Then

$$
\omega(T / S)=V N S(T)+1=n-2
$$

But $T(T /\{v\}) \leq n-2$, so $d(v)=1$ and $T / S$ is $n-2$ isolated vertices. Clearly, the star graph, $S_{1, n-1}$, is the unique tree in this case.

2) If $n \geq 7$ and $T$ is not isomorphic to $P_{n}$, then $\Delta(T) \geq 3$. We distinguish two cases.

Case 1: $\Delta(T)=3$.

Assume $u \in V(T)$ and $d(u)=3$, denote $N(u)=\{x, y, z\}$.

Case 1.1: If there are at least two vertices in $N(u)$ such that their degree are 3 . Then $\omega(T /\{u\}) \geq 4$. Thus $\operatorname{VNS}(T) \geq 3$.

Case 1.2: If there is unique vertex, say $x$, in $N(u)$ such that $d(x)=3$. Then $d(y) \leq 2, d(z) \leq 2$. We consider the following two possibilities.

Case 1.2.1: If both $d(y)$ and $d(z)$ are 2. Then $\omega(T /\{u\}) \geq 3, \operatorname{VNS}(T) \geq 2$.

Case 1.2.2: If both $d(y)$ and $d(z)$ are 1. Assume $N(x)-\{u\}=\{s, t\}$. Since $n \geq 7$, there is at least one vertex in $\{s, t\}$, say $s$, such that $d(s) \geq 2$. Thus $\omega(T /\{u\}) \geq 3$, and $\operatorname{VNS}(T) \geq 2$.

Case 1.3: The degree of $x, y$ and $z$ all are at most 2 . We discuss three possibilities. 
Case 1.3.1: If there is unique vertex, say $z$, such that $d(z)=2$. Since $n \geq 7$, clearly, $\omega(T /\{u\}) \geq 3$ and $\operatorname{VNS}(T) \geq 2$.

Case 1.3.2: If there are exact two vertices, say $y, z$, such that $d(y)=2, d(z)=2$. Since $n \geq 7$, we have $\omega(T /\{y\}) \geq 3$ or $\omega(T /\{z\}) \geq 3$. So $\operatorname{VNS}(T) \geq 2$.

Case 1.3.3: If $d(x)=d(y)=d(z)=2$, obviously $\omega(T /\{u\}) \geq 3$. So $\operatorname{VNS}(T) \geq 2$.

Case 2: $\Delta(T) \geq 4$.

Assume $d(u) \geq 4, v$ is any vertex adjacent to $u$. Then $\omega(T /\{v\}) \geq 3, \operatorname{VNS}(T) \geq 2$.

Therefore, we have $\operatorname{VNS}(T) \geq 2$ if $T \neq P_{n}$, contradicted to $\operatorname{VNS}(T)=1$. Thus $T=P_{n}$ and the proof is completed.

Remark 3: $P_{5}$ and $C_{3,2}$ are the two trees with order 5 such that $V N S=1$. There are six non-isomorphic trees with order 6 . The three trees with order 6 such that $V N S=1$ are shown in Figure 1.

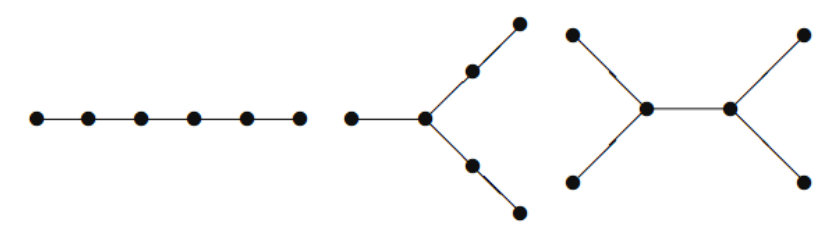

Figure 1. The trees of order 6 such that $V N S=1$.

\section{Acknowledgements}

This work was supported by the SXESF (Grant 09JK545) and the BSF (Grant JC0924).

\section{References}

[1] Z. Wei, A. Mai and M. Zhai, "Vertex-Neighbor-Scattering Number of Graphs," Ars Combinatoria, Vol. 102, 2011.

[2] F. Li and X. Li, "Computational Complexity and Bounds for Neighbor-Scattering Number of Graphs," 8th International Symposium on Parallel Architectures, Algorithms and Networks, Las Vegas, 7-9 December 2005, pp. 478-483. doi:10.1109/ISPAN.2005.30

[3] J. A. Bondy and U. S. R. Murty, "Graph Theory with Applications," Macmillan, London, 1976. 